\title{
SOLVABILITY OF INITIAL BOUNDARY VALUE PROBLEMS FOR EQUATIONS DESCRIBING MOTIONS OF LINEAR VISCOELASTIC FLUIDS
}

\author{
N. A. KARAZEEVA \\ Received 16 April 2003 and in revised form 28 July 2004
}

The nonlinear parabolic equations describing motion of incompressible media are investigated. The rheological equations of most general type are considered. The deviator of the stress tensor is expressed as a nonlinear continuous positive definite operator applied to the rate of strain tensor. The global-in-time estimate of solution of initial boundary value problem is obtained. This estimate is valid for systems of equations of any nonNewtonian fluid. Solvability of initial boundary value problems for such equations is proved under some additional hypothesis. The application of this theory makes it possible to prove the existence of global-in-time solutions of two-dimensional initial boundary value problems for generalized linear viscoelastic liquids, that is, for liquids with linear integral rheological equation, and for third-grade liquids.

\section{Introduction}

In the paper, we consider rheological equations of general type. We have got the global-intime estimate of solutions which is true for equations of motion of any non-Newtonian liquid in two-dimensional case. The additional condition imposed on the deviator of the stress tensor permits to obtain the weak solution of periodic initial boundary value problem and of the Cauchy problem. This condition is fulfilled for any linear viscoelastic fluid. Equations of motion of these fluids are approximations of the complete equations of motion of viscoelastic fluids. These equations were investigated in the monographs of Tschoegl [14] and of Golden et al. [6] and they have been of great interest in the recent years. We may point out the papers of Matei et al. [9], Fabrizio et al. [4], Gentili [5].

The theoretical possibility of application of linear viscoelastic equations is proved in the monography of Bird et al. [2]. The experimental verification of these equations is presented in many monographs. Among them I want to mention the monographs of Creus [3], Goodwin et al. [7] and Schwarzl [13].

Creus shows that the concrete structure is described by linear viscoelastic model. Goodwin and Hughes [7] presented many diagrams of correspondence of experimental data and of data calculated with the help of linear viscoelastic models for different 
materials (e.g., for polystyrene latex, polybutadiene, etc.). Schwarzl [13] presents the diagrams containing intervals of applications of linear viscoelastic models and the critical values for which the linearity is lost.

The solvability of initial boundary value problems for some special linear viscoelastic fluids was investigated by Oskolkov $[10,11,12]$. He proved the existence of global classical solutions of initial boundary value problems for linear viscoelastic Oldroyd equations in two-dimensional case and for linear viscoelastic Kelvin-Voight equations in threedimensional case. He did not consider linear viscoelastic equations of general type.

The motion of incompressible media is described by the system of equations

$$
\frac{\partial}{\partial t} v+v_{k} \frac{\partial v}{\partial x_{k}}+\operatorname{grad} p=\operatorname{div} \sigma+f, \quad \operatorname{div} v=0,
$$

where $\sigma=\left(\sigma_{i k}\right)$ is the deviator of the stress tensor, $\operatorname{tr} \sigma=0$ (by $\operatorname{tr} \sigma$ we denote the trace of the tensor $\sigma$ ). The type of the fluid is specified by the so-called rheological equation responding to state equation between $\sigma$ and the tensor of velocity deformations $D=(1 / 2)\left(v_{i x_{k}}+v_{k x_{i}}\right)$. This relation is called the rheological equation or the state equation. The simplest example of the state equation is the equation $\sigma=0$ which describes an ideal incompressible fluid. If we have the state equation of the type

$$
\sigma=2 \mu D
$$

we have a Newtonian fluid, and the motion is described by the Navier-Stokes equations.

In the present paper, we research different types of non-Newtonian fluids with memory. In the first part of the paper, we consider the rheological equation of the type

$$
\sigma=2 \mu D+\mathbf{K} D
$$

where the operator $\mathbf{K}$ is a continuous, positive-definite operator with some additional properties specified below. We consider the two-dimensional initial boundary value problem with nonslip boundary condition and prove the existence of a global strong solution. The difficulty consists of the fact that the term connected with the memory is nonlocal in time. Therefore, we can not use the estimates on the layers $\Omega$. All the obtained estimates are new.

It is shown that the equations of motion of general linear viscoelastic fluids with rheological equation of the type

$$
\sigma=2 \mu D+\int_{0}^{t} K(t-\tau) D(x, \tau) d \tau
$$

belong to this class. As a corollary, we apply the obtained results to the equations of motion of linear viscoelastic Oldroyd fluids. These are the equations which are obtained from the equations of motion of the Oldroyd fluids after substitution of the material derivatives by the ordinary partial derivatives. This substitution may be done in the case of small displacement gradients, which often occurs in the practice (see $[7,13]$ ).

Moreover, the results of this section permit to confirm the solvability of initial boundary value problems for equations of motion of third-grade fluids. Flows of these fluids are 
described by the rheological equations of the type

$$
\sigma=2 \mu D+\nu D^{3}
$$

If $\mu$ and $\nu$ are positive constants, then the operator in the right-hand side of (1.5) satisfies all the conditions imposed on the operator $\mathbf{K}$.

In the second part of the paper, we consider three-dimensional flows governed by the rheological equation

$$
\sigma=2 \mu_{1} D+2 \mathbf{K} D+2 \mu \frac{\partial D}{\partial t}
$$

with continuous, positive-definite operator $\mathbf{K}$. For this class of fluids, we investigate an initial boundary value problem with nonslip boundary condition. The existence of a strong solution is proved. Then we show that equations of motion of the linear viscoelastic Kelvin-Voight fluids belong to this class. So the existence of a strong global solution for three-dimensional initial boundary value problems for the linear viscoelastic KelvinVoight equations is proved.

In the third section, we investigate the most general rheological equation

$$
\sigma=\mathbf{K} D
$$

where the operator $\mathbf{K}$ is continuous and positive-definite. This equations correspond to arbitrary non-Newtonian (or Newtonian) fluid. We obtain the estimate of the solution of periodic initial boundary value problem in two-dimensional case. The requirement of positive definiteness makes a physical sense because the corresponding quadratic form is equal to

$$
-(\operatorname{div} \sigma, v)_{2, \Omega}=\int_{\Omega} \sigma: \nabla v=\int_{\Omega} \sigma: D
$$

which means that dissipation of energy is positive (see [1, Chapters 2-3]).

The two-dimensional Cauchy problem is investigated for these equations. We prove global solvability of it in the class of weak solutions under some additional hypothesis. Then we show that the equations of flows of general linear viscoelastic fluids, that is, flows generated by the linear rheological equations of general type

$$
\sigma=\int_{0}^{t} K(t-\tau) D(x, \tau) d \tau
$$

belong to this class of liquids, and thus we obtain the solvability theorem for the Cauchy problem for fluids of these types. As a corollary of these existence theorems, we prove the global-in-time existence of a weak solution in two-dimensional case for a system of equations of linear viscoelastic Maxwell fluids. 
62 Boundary value problems for linear viscoelastic fluids

\section{Notation}

In this section, we state preliminary mathematical notation. The symbol $\sigma: \sigma^{\prime}$ is used for the summation

$$
\sigma: \sigma^{\prime}=\operatorname{tr}\left(\sigma \sigma^{\prime}\right)=\sum_{i, k=1}^{n} \sigma_{i k} \sigma_{k i}^{\prime}
$$

We write $v_{t}$ for a partial derivative $\partial v / \partial t$, and we denote $\partial v / \partial x_{k}$ by $v_{x_{k}}$. The norm in the space $W_{2}^{l}(\Omega)$ is denoted by $\|u\|_{2, \Omega}^{l}$. Hilbert space $\stackrel{0}{W}_{2}^{l}$ is the closure of the set $\stackrel{0}{\mathbb{C}}^{\infty}$ in the norm $W_{2}^{l}$, where $\mathbb{C}^{\infty}$ is the set of infinitely differentiable functions with finite support. We write $\|u\|_{2, \infty}$ for the norm in the space $L_{2, \infty}$ :

$$
\|u\|_{2, \infty}=\sup _{t}\|u(x, t)\|_{2, \Omega}
$$

The norm $\|u\|_{2,1}$ in the space $L_{2,1}$ is defined as follows:

$$
\|u\|_{2,1}=\int_{0}^{t}\|u(x, t)\|_{2, \Omega} d t .
$$

By $\left\|u_{x}\right\|_{2, \Omega}$ we mean

$$
\left\|u_{x}\right\|_{2, \Omega}^{2}=\int_{\Omega} \sum_{k} u_{x_{k}}^{2}(x) d x
$$

and $\left(u_{x}, v_{x}\right)_{2, \Omega}$ is the corresponding scalar product

$$
\left(u_{x}, v_{x}\right)_{2, \Omega}=\int_{\Omega} \sum_{k} u_{x_{k}}(x) v_{x_{k}}(x) d x .
$$

Scalar product in the space $L_{2}(\Omega)$ is denoted by $(u, v)_{2, \Omega}$,

$$
(u, v)_{2, \Omega}=\int_{\Omega} u(x) v(x) d x
$$

The notation $\mathbf{L}_{2}, \mathbf{W}_{2}^{1}$ is used for the spaces of vector functions or tensors with components from the spaces $L_{2}$ or $W_{2}^{1}$ correspondingly. The space of Lipschitz continuous functions, that is, the space of functions with the finite norm

$$
\|u\|_{\mathbb{C}^{0,1}(\Omega)}=\sup _{x, y \in \Omega, x \neq y} \frac{|f(x)-f(y)|}{|x-y|}
$$

is denoted by $\mathbb{C}^{0,1}$. We use the notation $\mathbf{J}(\Omega)$ for the set of infinitely differentiable solenoidal vector functions with finite support

$$
\mathbf{J}(\boldsymbol{\Omega})=\left\{u \in \stackrel{0}{\mathbb{C}}^{\infty}(\Omega) \mid \operatorname{div} u=0\right\}
$$


The closure of this set in the $L_{2}$-norm is denoted by $\mathbf{J}^{0}(\Omega)$, and the closure in the $W_{2}^{1}$ norm is denoted by $\mathbf{H}(\Omega)$.

The subspace of all solenoidal fields in the space $\mathbf{W}_{2}^{1}(\Omega)$ which are periodic with respect to $x_{k}$ is denoted by $\hat{\mathbf{J}}_{2}^{1}$. The closure of this space in the $L_{2}(\Omega)$-norm is denoted by $\widehat{\mathbf{J}}_{2}^{0}(\Omega)$.

In the paper, we use some well-known inequalities. For the sake of convenience, we list them here.

(1) The Young inequality,

$$
a b \leq \frac{1}{\lambda} \epsilon^{\lambda} a^{\lambda}+\frac{1}{\lambda^{\prime}} \epsilon^{-\lambda^{\prime}} b^{\lambda^{\prime}},
$$

where $a, b, \epsilon$ are arbitrary positive numbers, $\lambda$ and $\lambda^{\prime}$ are more than unit, and $(1 / \lambda)+\left(1 / \lambda^{\prime}\right)=1$.

(2) Inequalities for the norm $\|u\|_{4, \Omega}$ (Ladyzhenskaya [8, Chapter 1]).

For every function $u \in \stackrel{0}{W}_{2}^{1}(\Omega), \Omega \subset \mathbb{R}^{2}$, it holds that

$$
\|u\|_{4, \Omega}^{4} \leq 2\|u\|_{2, \Omega}^{2}\left\|u_{x}\right\|_{2, \Omega}^{2}
$$

and for every function $u(x) \in \stackrel{0}{W}_{2}^{1}(\Omega), \Omega \subset \mathbb{R}^{3}$, it holds that

$$
\|u\|_{4, \Omega}^{4} \leq\left(\frac{4}{3}\right)^{3 / 2}\|u\|_{2, \Omega}\left\|u_{x}\right\|_{2, \Omega}^{3}
$$

(3) The Gronwall lemma.

Let a nonnegative absolutely continuous function $y(t)$ satisfy the inequality

$$
\frac{d y}{d t} \leq c_{1}(t) y(t)+c_{2}(t)
$$

for a.e. $t \in[0, T]$, where $c_{1}, c_{2} \in L_{1}[0, T]$ are nonnegative. Then,

$$
y(t) \leq \exp \left(\int_{0}^{t} c_{1}(\tau) d \tau\right) \cdot\left[y(0)+\int_{0}^{t} c_{2}(\tau) d \tau\right] .
$$

\section{Generalized equations of motion of viscous fluids}

3.1. Statement of the problem. We consider the following system of equations:

$$
\frac{\partial v}{\partial t}+v_{k} \frac{\partial v}{\partial x_{k}}-\mu \Delta v-\operatorname{div}(\mathbf{K} D)+\operatorname{grad} p=f, \quad \operatorname{div} v=0 .
$$

The system is studied in a bounded domain $\Omega \subset \mathbb{R}^{2}, Q_{T}=\Omega \times[0, T), T \in(0, \infty], v=$ $\left(v_{1}, v_{2}\right): Q_{T} \rightarrow \mathbb{R}^{2}$ is a velocity field, $p: Q_{T} \rightarrow \mathbb{R}$ is a pressure, and $f: Q \rightarrow \mathbb{R}^{2}$ is the field of external forces. The boundary $\partial \Omega$ is supposed to satisfy some smoothness condition. This condition should be enough to provide the embedding theorems. It is sufficient to assume that $\partial \Omega$ is Lipschitz continuous. Let the operator $\mathbf{K}: \mathbf{L}_{2}\left(Q_{T}\right) \rightarrow \mathbf{L}_{2}\left(Q_{T}\right)$ be continuous and satisfy the following conditions: 
64 Boundary value problems for linear viscoelastic fluids

(1) $\mathrm{K}$ is bounded, that is,

$$
\|\mathbf{K} D\|_{2, Q_{T}} \leq c_{k}\|D\|_{2, Q_{T}} .
$$

(2) $\mathbf{K}$ is positive-definite in the following sense:

$$
\int_{Q_{T}}((\mathbf{K} D): D) \geq 0
$$

(3) the operator $(\partial / \partial t) \circ \mathbf{K}$ is bounded in the space $\mathbf{L}_{2}\left(Q_{T}\right)$, that is,

$$
\left\|\frac{\partial}{\partial t}(\mathbf{K} D)\right\|_{2, Q_{T}} \leq c_{1}\|D\|_{2, Q_{T}} .
$$

We consider the initial boundary value problem

$$
\left.v\right|_{t=0}=v_{0}(x),\left.\quad v\right|_{\partial Q_{T}}=0 .
$$

By $V\left(Q_{T}\right)$ we denote the space of vector functions $v$ equipped with the norm

$$
[v]_{Q_{T}}=\sup _{0 \leq t \leq T}\|v(x, t)\|_{2, \Omega}+\left\|v_{x}\right\|_{2, Q_{T}} .
$$

We introduce the notion of generalized solution of the problem (3.1)-(3.5); the function $v \in V\left(Q_{T}\right)$ which satisfies the integral identity

$$
\begin{gathered}
\int_{Q_{T}}\left(-v \phi_{t}-v_{k} v \phi_{x_{k}}+\mu v_{x} \phi_{x}+(\mathbf{K} D) \nabla \phi\right) d x d t+\left.\int_{\Omega} v \phi\right|_{t=T} d x-\left.\int_{\Omega} v_{0} \phi\right|_{t=0} d x \\
=\int_{Q_{T}} f \phi d x d t
\end{gathered}
$$

for any $\phi \in \stackrel{\mathbf{W}}{2}_{2}^{1,1} \cap \mathbf{J}\left(Q_{T}\right)$.

To verify the correctness of the definition, it is necessary to prove the finiteness of the integral $\int_{Q_{T}}(\mathbf{K} D) \nabla \phi$,

$$
\left|\int_{Q_{T}}(\mathbf{K} D) \nabla \phi\right| \leq\|\mathbf{K} D\|_{2, Q_{T}}\left\|\phi_{x}\right\|_{2, Q_{T}} \leq c_{k}\left\|v_{x}\right\|_{2, Q_{T}}\left\|\phi_{x}\right\|_{2, Q_{T}} .
$$

3.2. A priori estimates. In order to obtain some a priori estimates for solutions, we multiply the system by $v$ and integrate over $\Omega$. After integrating by parts, we arrive at the identity

$$
\frac{1}{2} \frac{d}{d t}\|v\|_{2, \Omega}^{2}+\mu\left\|v_{x}\right\|_{2, \Omega}^{2}+\int_{\Omega}((\mathbf{K} D): D)=\int_{\Omega} f v
$$

Integration with respect to $t$ on $[0, t]$ yields

$$
\frac{1}{2}\left(\|v(t)\|_{2, \Omega}^{2}-\|v(0)\|_{2, \Omega}^{2}\right)+\mu \int_{0}^{t}\left\|v_{x}\right\|_{2, \Omega}^{2}+\int_{Q_{T}}((\mathbf{K} D): D)=\int_{Q_{t}} f v .
$$


By the nonnegativity of terms (3.3), the following estimate holds:

$$
\begin{aligned}
& \|v(t)\|_{2, \Omega}^{2} \leq\|v(0)\|_{2, \Omega}^{2}+2 \int_{0}^{t}\left|\int_{\Omega} f v\right|, \\
& \|v(t)\|_{2, \Omega}^{2} \leq\|v(0)\|_{2, \Omega}^{2}+2 \int_{0}^{t}\|f\|_{2, \Omega}\|v\|_{2, \Omega}, \\
& \|v(t)\|_{2, \Omega}^{2} \leq\|v(0)\|_{2, \Omega}^{2}+\|v\|_{2, \infty} \int_{0}^{t}\|f\|_{2, \Omega} .
\end{aligned}
$$

After maximization of the left-hand side, we obtain the inequality

$$
\|v\|_{2, \infty}^{2} \leq\|v(0)\|_{2, \Omega}^{2}+2\|v\|_{2, \infty} \int_{0}^{t}\|f\|_{2, \Omega} .
$$

Solving this quadratic inequality, we get an estimate for the norm of $v$ :

$$
\|v\|_{2, \infty} \leq \int_{0}^{t}\|f\|_{2, \Omega}+\sqrt{\left(\int_{0}^{t}\|f\|_{2, \Omega}\right)^{2}+\|v(0)\|_{2, \Omega}^{2}}:=c_{2}(t) .
$$

Moreover, (3.10) leads to the estimate

$$
\frac{1}{2}\left(\|v(t)\|_{2, \Omega}^{2}-\|v(0)\|_{2, \Omega}^{2}\right)+\mu \int_{0}^{t}\left\|v_{x}\right\|_{2, \Omega}^{2} \leq \int_{0}^{t}\left|\int_{\Omega} f v\right| .
$$

Consequently,

$$
\begin{aligned}
\|v(t)\|_{2, \Omega}^{2}+2 \mu \int_{0}^{t}\left\|v_{x}\right\|_{2, \Omega}^{2} & \leq 2 \int_{0}^{t}\left|(f, v)_{\Omega}\right|+\|v(0)\|_{2, \Omega}^{2} \\
& \leq\|v(0)\|_{2, \Omega}^{2}+2 \int_{0}^{t}\|f\|_{2, \Omega}\|v\|_{2, \Omega}, \\
\|v(t)\|_{2, \Omega}^{2}+2 \mu \int_{0}^{t}\left\|v_{x}\right\|_{2, \Omega}^{2} & \leq\|v(0)\|_{2, \Omega}^{2}+2\|v\|_{2, \infty} \int_{0}^{t}\|f\|_{2, \Omega} \\
& \leq\|v(0)\|_{2, \Omega}^{2}+2\|f\|_{2,1}\left(\|f\|_{2,1}+\sqrt{\|f\|_{2,1}^{2}+\|v(0)\|_{2, \Omega}^{2}}\right) \\
& :=c_{3}(t) .
\end{aligned}
$$

Now we differentiate the first equation of system (3.1) with respect to $t$, multiply by $v_{t}$, and integrate over $\Omega$. Integration by parts yields

$$
\frac{1}{2} \frac{d}{d t}\left\|v_{t}\right\|_{2, \Omega}^{2}+\mu\left\|v_{x t}\right\|_{2, \Omega}^{2}-\int_{\Omega} \frac{\partial}{\partial t}(\mathbf{K} D) \nabla v_{t}+\int_{\Omega} v_{k t} v_{x_{k}} v_{t}=\left(f_{t}, v_{t}\right)
$$

By the Cauchy inequality, we have

$$
\left|\int_{\Omega} v_{k t} v_{x_{k}} v_{t}\right| \leq\left\|v_{x}\right\|_{2, \Omega} \cdot\left\|v_{t}\right\|_{4, \Omega}^{2}
$$


The terms in the right-hand side may be estimated with the help of (2.10) in twodimensional case.

$$
\left|\int_{\Omega} v_{k t} v_{x_{k}} v_{t}\right| \leq \sqrt{2}\left\|v_{x}\right\|_{2, \Omega} \cdot\left\|v_{t}\right\|_{2, \Omega} \cdot\left\|v_{x t}\right\|_{2, \Omega}
$$

By virtue of the Young inequality, it holds that

$$
\left|\int_{\Omega} v_{k t} v_{x_{k}} v_{t}\right| \leq \sqrt{2}\left(\kappa_{2}\left\|v_{x t}\right\|_{2, \Omega}^{2}+c_{\kappa_{2}}\left\|v_{x}\right\|_{2, \Omega}^{2}\left\|v_{t}\right\|_{2, \Omega}^{2}\right)
$$

where $c_{\mathcal{K}_{2}}=(1 / 4) \kappa_{2}^{-1}, \kappa_{2}$ is a positive number which will be chosen later.

Due to the Young inequality, the other terms of (3.16) are estimated as follows:

$$
\begin{aligned}
\left|\left(f_{t}, v_{t}\right)_{2, \Omega}\right| & \leq\left\|f_{t}\right\|_{2, \Omega}\left\|v_{t}\right\|_{2, \Omega} \leq \frac{1}{2}\left\|f_{t}\right\|_{2, \Omega}+\frac{1}{2}\left\|f_{t}\right\|_{2, \Omega}\left\|v_{t}\right\|_{2, \Omega}^{2}, \\
\left|\int_{\Omega} \frac{\partial}{\partial t}(\mathbf{K} D) \nabla v_{t}\right| & \leq\left\|\frac{\partial}{\partial t} \mathbf{K} D\right\|_{2, \Omega}\left\|v_{x t}\right\|_{2, \Omega} \leq \kappa_{1}\left\|v_{x t}\right\|_{2, \Omega}^{2}+c_{\kappa_{1}}\left\|\frac{\partial}{\partial t}(\mathbf{K} D)\right\|_{2, \Omega}^{2},
\end{aligned}
$$

where $c_{\kappa_{1}}=(1 / 4) \kappa_{1}^{-1}$.

We set $\sqrt{2} \kappa_{2}=\mu / 4$ and $\kappa_{1}=\mu / 4$. Then we get

$$
\begin{aligned}
\frac{1}{2} \frac{d}{d t}\left\|v_{t}\right\|_{2, \Omega}^{2}+\mu\left\|v_{x t}\right\|_{2, \Omega}^{2} \leq & \left(\frac{1}{2}\left\|f_{t}\right\|_{2, \Omega}+\frac{1}{2}\left\|f_{t}\right\|_{2, \Omega}\left\|v_{t}\right\|_{2, \Omega}^{2}\right) \\
& +\left(\frac{\mu}{4}\left\|v_{x t}\right\|_{2, \Omega}^{2}+\frac{2}{\mu}\left\|v_{x}\right\|_{2, \Omega}^{2}\left\|v_{t}\right\|_{2, \Omega}^{2}\right) \\
& +\left(\frac{\mu}{4}\left\|v_{x t}\right\|_{2, \Omega}^{2}+\frac{1}{\mu}\left\|\frac{\partial}{\partial t} \mathbf{K} D\right\|_{2, \Omega}^{2}\right) .
\end{aligned}
$$

Consequently,

$$
\frac{1}{2} \frac{d}{d t}\left\|v_{t}\right\|_{2, \Omega}^{2} \leq \frac{1}{2}\left\|f_{t}\right\|_{2, \Omega}+\frac{1}{2}\left\|f_{t}\right\|_{2, \Omega}\left\|v_{t}\right\|_{2, \Omega}^{2}+\frac{2}{\mu}\left\|v_{x}\right\|_{2, \Omega}^{2}\left\|v_{t}\right\|_{2, \Omega}^{2}+\frac{1}{\mu}\left\|\frac{\partial}{\partial t}(\mathbf{K} D)\right\|_{2, \Omega}^{2} .
$$

By the Gronwall lemma (2.13),

$$
\begin{aligned}
\left\|v_{t}\right\|_{2, \Omega}^{2} \leq & \exp \left(\int_{0}^{t}\left(\left\|f_{t}\right\|_{2, \Omega}+\frac{4}{\mu}\left\|v_{x}\right\|_{2, \Omega}^{2}\right) d \tau\right) \\
& \cdot\left[\left\|v_{t}(0)\right\|_{2, \Omega}^{2}+\int_{0}^{t}\left(\left\|f_{t}\right\|_{2, \Omega}+\frac{1}{\mu}\left\|\frac{\partial}{\partial t} \mathbf{K} D\right\|_{2, \Omega}^{2}\right) d \tau\right] .
\end{aligned}
$$

Since $\int_{0}^{t}\|(\partial / \partial t) \mathbf{K} D\|_{2, \Omega}^{2} \leq c_{1}^{2}\left\|v_{x}\right\|_{2, Q_{T}}^{2}$ (3.4) and $2 \mu\left\|v_{x}\right\|_{2, Q_{T}}^{2} \leq c_{3}$ (3.15), the following estimate is true for norm $\left\|v_{t}\right\|_{2, \Omega}^{2}$ :

$$
\begin{aligned}
\left\|v_{t}\right\|_{2, \Omega}^{2} \leq & \exp \left(\left(\int_{0}^{t}\left\|f_{t}\right\|_{2, \Omega}\right)+\frac{2}{\mu^{2}} c_{3}(t)\right) \\
& \cdot\left[\left\|v_{t}(0)\right\|_{2, \Omega}^{2}+\left(\int_{0}^{t}\left\|f_{t}\right\|_{2, \Omega}\right)+\frac{1}{2 \mu^{2}} c_{1}^{2} c_{3}\right]:=c_{4}(t)
\end{aligned}
$$


Moreover, by inequality (3.21), one can estimate $\int_{0}^{t}\left\|v_{x t}\right\|_{2, \Omega}^{2}$. Indeed, by integrating (3.21) with respect to $t$ on $[0, t]$ we obtain

$$
\begin{aligned}
\left\|v_{t}(t)\right\|_{2, \Omega}^{2}+\mu \int_{0}^{t}\left\|v_{x t}\right\|_{2, \Omega}^{2} \leq & \left\|v_{t}(0)\right\|_{2, \Omega}^{2} \\
& +\int_{0}^{t}\left\|f_{t}\right\|_{2, \Omega}+\left(\int_{0}^{t}\left\|f_{t}\right\|_{2, \Omega}\right)\left\|v_{t}\right\|_{2, \infty}^{2} \\
& +\frac{4}{\mu}\left\|v_{t}\right\|_{2, \infty}^{2} \int_{0}^{t}\left\|v_{x}\right\|_{2, \Omega}^{2}+\frac{2}{\mu} \int_{0}^{t}\left\|\frac{\partial}{\partial t} \mathbf{K} D\right\|_{2, \Omega}^{2} d \tau,\left\|v_{t}(t)\right\|_{2, \Omega}^{2} \\
& +\mu \int_{0}^{t}\left\|v_{x t}\right\|_{2, \Omega}^{2} \leq\left\|v_{t}(0)\right\|_{2, \Omega}^{2}+c_{4}(t) \int_{0}^{t}\left\|f_{t}\right\|_{2, \Omega} \\
& +\int_{0}^{t}\left\|f_{t}\right\|_{2, \Omega}+\frac{2}{\mu^{2}} c_{3}(t) c_{4}(t)+\frac{1}{\mu^{2}} c_{1}^{2} c_{3}=c_{5}(t) .
\end{aligned}
$$

3.3. Existence of solutions. The estimates (3.15), (3.24), and (3.25) obtained in Section 3.2 can be used for the proof of the existence theorems for problem (3.1)-(3.5).

THEOREM 3.1. Let $\Omega$ be an arbitrary bounded domain in $\mathbb{R}^{2}, \partial \Omega \in \mathbb{C}^{0,1}$,

$$
f, f_{t} \in \mathbf{L}_{2,1}\left(Q_{T}\right), \quad v_{0} \in \mathbf{W}_{2}^{2}(\Omega) \cap \mathbf{J}^{0}(\Omega), \quad 0<T \leq \infty .
$$

Let the operator $\mathbf{K}$ satisfy conditions (3.2)-(3.4). Then problem (3.1)-(3.5) has a generalized solution

$$
v \in \mathbf{L}_{\infty}\left(0, T ; \mathbf{J}^{0}(\Omega)\right), \quad v_{t} \in \mathbf{L}_{\infty}\left(0, T ; \mathbf{J}^{0}(\Omega)\right) .
$$

Moreover, $v_{x} \in \mathbf{L}_{2}\left(Q_{T}\right)$ and $v_{x t} \in \mathbf{L}_{2}\left(Q_{T}\right)$, and the following estimates hold:

$$
\begin{gathered}
2 \mu \int_{0}^{t}\left\|v_{x}\right\|_{2, \Omega}^{2}+\left\|v_{x}\right\|_{2, \infty}^{2} \leq c_{3}\left(\|f\|_{2,1} ;\|v(0)\|_{2, \Omega}\right), \\
\mu \int_{0}^{t}\left\|v_{x t}\right\|_{2, \Omega}^{2}+\left\|v_{t}\right\|_{2, \Omega}^{2} \leq c_{5}\left(\left\|f_{t}\right\|_{2,1} ;\|v(0)\|_{2, \Omega}\right),
\end{gathered}
$$

furthermore, $c_{3}(t), c_{5}(t)$ tend to some constants $c_{3}, c_{5}<\infty$ for $t \rightarrow \infty$.

The corollary of this theorem is the existence of smooth global-in-time solution for equations of motion of general linear viscoelastic fluids with rheological equation of the type (1.4) in the case when the inverse Fourier transform of the function $K$ is positive. The other corollary consists of existence of smooth global-in-time solution of initial boundary value problem for third-grade fluids governed by the rheological equation of the type (1.5).

Proof. To prove this theorem we can use the Galerkin method. Let $\left\{\varphi^{k}\right\}, k=1,2, \ldots$, be a complete system of functions in $\mathbf{W}_{2}^{2}(\Omega) \cap \mathbf{H}(\Omega)$, which is orthonormal in $\mathbf{L}_{2}(\Omega)$. 
Then for every $v_{0} \in \mathbf{W}_{2}^{2}(\Omega) \cap \mathbf{H}(\Omega)$, there is a sequence of functions

$$
v_{(n)}^{0}(x)=\sum_{k=1}^{n} c_{k n}^{0} \varphi^{k}(x)
$$

such that

$$
\left\|v_{(n)}^{0}(x)-v_{0}\right\|_{W_{2}^{2}(\Omega)} \longrightarrow 0
$$

An approximate solution of problem (3.1)-(3.5) can be represented in the form

$$
v^{n}(x, t)=\sum_{k=1}^{n} c_{k n}(t) \varphi^{k}(x), \quad n=1,2, \ldots
$$

where the functions $c_{k n}$ satisfy the following system of integral identities:

$$
\left(v_{t}^{n}, \varphi^{k}\right)+\mu\left(v_{x}^{n}, \varphi_{x}^{k}\right)-\left(v_{i}^{n} v^{n}, \varphi_{x_{i}}^{k}\right)+\left(\mathbf{K} \nabla v^{n}, \nabla \varphi^{k}\right)=\left(f, \varphi^{k}\right),
$$

and the initial conditions:

$$
\left.c_{k n}\right|_{t=0}=c_{k n}^{0}, \quad k=1, \ldots, n \text {. }
$$

These identities form the system of ordinary differential equations with respect to the functions $c_{n k}$,

$$
\frac{d c_{k n}}{d t}+\mu \sum_{i=1}^{n} \varphi_{k i} c_{i n}(t)+\sum_{i, j=1}^{n} \varphi_{k i j} c_{i n}(t) c_{j n}(t)+\sum_{i=1}^{n}\left(\mathbf{K}\left(\nabla \varphi_{i}(x) c_{i n}(t)\right), \nabla \varphi^{k}\right)=f_{k}(t),
$$

where $\varphi_{k i}, \varphi_{k i j}$ are the constants

$$
\varphi_{k i}=\left(\varphi_{x}^{i}, \varphi_{x}^{k}\right)_{2, \Omega}, \quad \varphi_{k i j}=\sum_{l=1}^{n} \int_{\Omega} \varphi_{l}^{j} \varphi^{i} \varphi_{x_{l}}^{k}, \quad f_{k}=\left(f, \varphi^{k}\right)_{2, \Omega} .
$$

System (3.34) is a system of ordinary differential equations in the normal form and may be solved, for instance, by the method of Picard iterations.

To prove the theorem, we show that the approximations $v^{n}$ satisfy estimates (3.15), (3.25). Indeed $v_{n}$ satisfy the integral identities (3.9) and (3.16). Identity (3.16) can be obtained in the following way: identities (3.32) are differentiated with respect to $t$, then multiplied by $(d / d t) c_{k n}$, and summarized with respect to $k \in\{1, \ldots, n\}$.

Estimates (3.15), (3.25) and the theorem of weak compactness of bounded sets in Hilbert spaces permit to choose a convergent subsequence $\left\{v^{n_{k}}\right\}$ from the sequence $v^{n}$. The functions $v^{n_{k}}, v_{x}^{n_{k}}, v_{t}^{n_{k}}, v_{x t}^{n_{k}}$ converge to $v, v_{x}, v_{t}, v_{x t}$, respectively, in the norm $\mathrm{L}_{2}\left(Q_{T}\right)$.

To prove the solvability of the problem, it is necessary to verify that the function $v$ satisfies the integral identity (3.7). This follows from the possibility of the limit passage in identities (3.32). 
3.4. Equations of motion of the Oldroyd fluids. We now prove that the linear viscoelastic Oldroyd flow of order $L=1,2, \ldots$, in a bounded two-dimensional domain $\Omega$ is a flow of considered type. This flow is described by the system of equations

$$
\frac{\partial v}{\partial t}+v_{k} \frac{\partial v}{\partial x_{k}}+\operatorname{grad} p=\operatorname{div} \sigma+f, \quad \operatorname{div} v=0,
$$

where $\sigma$ is the deviator of the stress tensor. For fluids of Oldroyd type, this tensor is connected with the tensor of velocity deformations by the relation

$$
\left(1+\sum_{l=1}^{L} \lambda_{l} \frac{\partial^{l}}{\partial t^{l}}\right) \sigma=2 \nu\left(1+\sum_{m=1}^{M} \kappa_{m} \nu^{-1} \frac{\partial^{m}}{\partial t^{m}}\right) D .
$$

Here $\lambda_{l}>0$ are the relaxation times, and $\kappa_{m}>0$ are the retardation times, and $L=M$. We apply the Laplace transform $\mathscr{L}$ to both parts of (3.37)

$$
\mathscr{L} \sigma=\hat{\sigma}(x, p)=\int_{0}^{\infty} e^{-p t} \sigma(x, t) d t .
$$

Consider polynomial $Q(p)=1+\sum_{l=1}^{L} \lambda_{l} p^{l}$. Then it holds

$$
\hat{\sigma}(x, p)=2 Q^{-1}(p)\left(\nu+\sum_{l=1}^{M} \kappa_{l} p^{l}\right) \hat{D}(x, p) .
$$

To obtain $\sigma$ in explicit form, we apply the inverse Laplace transform $\mathscr{L}^{-1}$ to (3.39).

$$
\sigma(x, t)=2 \int_{0}^{t} G(t-\tau) D(x, \tau) d \tau,
$$

where

$$
G(t)=\mathscr{L}^{-1}\left(Q^{-1}(p)\left(\nu+\sum_{l=1}^{M} \kappa_{l} p^{l}\right)\right) .
$$

By introduction of the polynomial $P_{o}(p)$,

$$
P_{o}(p)=\nu-\mu+\sum_{l=1}^{L}\left(\kappa_{l}-\mu \lambda_{l}\right) p^{l}
$$

where $\mu=\kappa_{l} \lambda_{l}^{-1}$, we arrive at the expressions

$$
\begin{aligned}
& G(t)=\mathscr{L}^{-1}\left(\mu+Q^{-1}(p) P_{o}(p)\right), \\
& G(t)=\mu \delta(t)+\sum_{s=1}^{L} \frac{P_{o}\left(-\alpha_{s}\right)}{Q^{\prime}\left(-\alpha_{s}\right)} e^{-\alpha_{s} t}, \\
& G(t)=\mu \delta(t)+\sum_{s=1}^{L} \beta_{s}^{(o)} e^{-\alpha_{s} t},
\end{aligned}
$$


where $\delta(t)$ is the Dirac $\delta$-function, $\left(-\alpha_{s}\right)$ are the roots of the polynomial $Q(p)$, and

$$
\beta_{s}^{(o)}=P_{o}\left(-\alpha_{s}\right)\left(Q^{\prime}\left(-\alpha_{s}\right)\right)^{-1}
$$

Suppose that the roots $-\alpha_{s}$ are single, that is, $Q^{\prime}\left(-\alpha_{s}\right) \neq 0, l=1, \ldots, L$, real-valued and negative. The coefficients $\beta_{s}^{(o)}$ are supposed to be positive.

After substituting (3.43) into (3.40), we get

$$
\sigma=\mu D+\sum_{s=1}^{L} \beta_{s}^{(o)} \int_{0}^{t} e^{-\alpha_{s}(t-\tau)} D d \tau
$$

In this way, the equations of motion of the Oldroyd fluids take the form

$$
\frac{\partial v}{\partial t}+v_{k} \frac{\partial v}{\partial x_{k}}-\mu \Delta v-\int_{0}^{t} \sum_{s=1}^{L} \beta_{s}^{(o)} e^{-\alpha_{s}(t-\tau)} \Delta v(x, \tau) d \tau+\operatorname{grad} p=f, \quad \operatorname{div} v=0 .
$$

This is the system of type (3.1) with the operator

$$
\mathbf{K}_{o} v=\int_{0}^{t} K(t-\tau) v(x, \tau) d \tau
$$

with the kernel $K$

$$
K(t)=\sum_{s=1}^{L} \beta_{s}^{(o)} e^{-\alpha_{s}(t)}
$$

We should prove that the operator $\mathbf{K}_{o}$ satisfies conditions (3.2)-(3.4).

Indeed, from the properties of the integral operators, it follows that the operator $\mathbf{K}_{o}$ is continuous in the space $\mathbf{L}_{2}\left(Q_{T}\right)$, and its norm does not exceed

$$
c_{k}=\sqrt{\left(\sup _{0 \leq t \leq T} \int_{0}^{t} K(\tau) d \tau\right)\left(\sup _{0 \leq \tau \leq T} \int_{0}^{T-\tau} K(t) d t\right)}, \quad c_{k}=\int_{0}^{T} K(t) d t .
$$

Then we prove that the quadratic form $\int_{Q_{T}}(\mathbf{K} v) v$ is positive definite. This fact follows from the following lemma.

Lemma 3.2. For every $u(\tau) \in L_{2}([0, t])$, it holds that

$$
I=\int_{0}^{t} \int_{0}^{\sigma} K(\sigma-\tau) u(\sigma) u(\tau) d \sigma d \tau \geq 0
$$

Proof. Suppose that the function $K$ is continued on the negative semiaxis like an even function. Then $I$ is equal to the half of integral on $[0, t] \times[0, t]$. If we extend $u$ by zero to $\mathbb{R}$ and denote the obtained function by $\tilde{u}$, we may rewrite $I$ in the form

$$
\frac{1}{2} \int_{-\infty}^{\infty} \int_{-\infty}^{\infty} K(\sigma-\tau) \tilde{u}(\sigma) \tilde{u}(\tau) d \sigma d \tau
$$


To prove that the integral (3.51) is positive for every $u \in L_{2}(\mathbb{R})$, we calculate at first the inverse Fourier transform of the function $K$ :

$$
\mathscr{F}^{-1}(K)=L(p)=(2 \pi)^{-1 / 2} \sum_{s=1}^{L} \frac{2 \beta_{s} \alpha_{s}}{p^{2}+\alpha_{s}^{2}} \geq 0 .
$$

Then we transform $I$ :

$$
\begin{aligned}
& I=\frac{1}{2} \int_{-\infty}^{\infty} \int_{-\infty}^{\infty} K(\sigma-\tau) \tilde{u}(\sigma) \tilde{u}(\tau) d \sigma d \tau, \\
& I=\frac{1}{2} \int_{-\infty}^{\infty} \int_{-\infty}^{\infty} \mathscr{F}(L)(\sigma-\tau) \tilde{u}(\sigma) \tilde{u}(\tau) d \sigma d \tau, \\
& I=(2 \pi)^{-1 / 2} \iint_{\mathbb{R}^{3}} \int e^{-i(\sigma-\tau) p} L(p) d p \tilde{u}(\sigma) \tilde{u}(\tau) d \sigma d \tau, \\
& I=\int_{\mathbb{R}}\left|\int_{\mathbb{R}} e^{-i \sigma p} \tilde{u}(\sigma) d \sigma\right|^{2} L(p) d p \geq 0 .
\end{aligned}
$$

The lemma is proved.

We see that condition (3.3) is fulfilled. To verify condition (3.4), transform the corresponding relation:

$$
\left\|\frac{\partial}{\partial t}(\mathbf{K} D)\right\|_{2, Q_{T}}=\left\|\frac{\partial}{\partial t} \int_{0}^{t} K(t-\tau) D(x, \tau) d \tau\right\|_{2, Q_{T}} \leq\|K(0) D(x, t)\|_{2, Q_{T}}+\left\|\mathbf{K}_{o}^{\prime} D\right\|_{2, Q_{T}},
$$

where $\mathbf{K}_{o}^{\prime}$ is an integral operator of Volterra type with the kernel $(d / d t) K$ :

$$
\left(\mathbf{K}_{o}^{\prime} u\right)(t)=\int_{0}^{t} \frac{d}{d t} K(t-\tau) u(\tau) d \tau
$$

The operator $\mathbf{K}_{o}^{\prime}$ is continuous in the space $\mathbf{L}_{2}\left(Q_{t}\right)$, and

$$
\int_{0}^{t}\left\|\mathbf{K}_{o}^{\prime} v_{x}\right\|_{2, \Omega}^{2} \leq c_{k}^{\prime} \int_{0}^{t}\left\|v_{x}\right\|_{2, \Omega}^{2}
$$

Thus $\|(\partial / \partial t)(\mathbf{K} D)\|_{2, Q_{T}}$ can be estimated in the following way:

$$
\left\|\frac{\partial}{\partial t}(\mathbf{K D})\right\|_{2, Q_{T}} \leq K(0)\left\|v_{x}\right\|_{2, Q_{T}}+c_{k}^{\prime}\left\|v_{x}\right\|_{2, Q_{T}} \leq \frac{1}{2 \mu}\left(K(0)+c_{k}^{\prime}\right) c_{3}:=c_{1} .
$$

So we proved that the equation, which describes motion of the Oldroyd fluids, satisfies the condition (3.2)-(3.4). Consequently, the corresponding initial boundary value problem admits a solution. 
72 Boundary value problems for linear viscoelastic fluids

\section{Equations of motion of generalized Kelvin-Voight fluids}

4.1. The abstract problem. Consider a PDE system of the following type:

$$
\frac{\partial v}{\partial t}+v_{k} \frac{\partial v}{\partial x_{k}}-\mu \frac{\partial \Delta v}{\partial t}-\mu_{1} \Delta v-\operatorname{div}(\mathbf{K} D)+\operatorname{grad} p=f, \quad \operatorname{div} v=0, \mu, \mu_{1}>0 .
$$

The system is considered in the cylinder $Q_{T}=\Omega \times[0, T), 0<T \leq \infty, \Omega$ is a bounded three-dimensional domain. Suppose that the operator K satisfies conditions (3.2)-(3.4) and that the solution of the system satisfies the following initial boundary conditions:

$$
\left.v\right|_{t=0}=v_{0}(x),\left.\quad v\right|_{\partial Q_{T}}=0 .
$$

We introduce the notion of generalized solution. A function $v \in \mathbf{V}\left(Q_{T}\right)$ is called the generalized solution if it satisfies the integral identity

$$
\begin{aligned}
\int_{Q_{T}}( & \left.-v \phi_{t}-v_{k} v \phi_{x_{k}}+\mu_{1} v_{x} \phi_{x}-\mu v_{x} \phi_{x t}+(\mathbf{K} D) \nabla \phi_{x}\right) d x d t \\
& +\left.\int_{\Omega}\left(v \phi-\mu v_{x} \phi_{x}\right)\right|_{t=T} d x-\left.\int_{\Omega}\left(v_{0} \phi-\mu v_{x} \phi_{x}\right)\right|_{t=0} d x=\int_{Q_{T}} f \phi
\end{aligned}
$$

for any $t \in[0, T]$ and for any test function $\phi \in \stackrel{0}{\mathbf{W}}_{2}^{1,1}\left(Q_{T}\right) \cup \mathbf{J}\left(Q_{T}\right)$.

4.2. A priori estimates. To obtain some a priori estimates for solutions of initial boundary value problem (4.1)-(4.2), we multiply the first equation of system (4.1) by $v$ and integrate with respect to $x$.

$$
\frac{1}{2} \frac{d}{d t}\|v\|_{2, \Omega}^{2}+\mu_{1}\left\|v_{x}\right\|_{2, \Omega}^{2}+(\mathbf{K} D, \nabla v)+\frac{\mu}{2} \frac{d}{d t}\left\|v_{x}\right\|_{2, \Omega}^{2}=(f, v) .
$$

Integrating system (4.4) again with respect to $t$ over the interval $[0, t)$, we get

$$
\begin{gathered}
\frac{1}{2}\left(\|v(t)\|_{2, \Omega}^{2}-\|v(0)\|_{2, \Omega}^{2}\right)+\frac{1}{2} \mu\left(\left\|v_{x}(t)\right\|_{2, \Omega}^{2}-\left\|v_{x}(0)\right\|_{2, \Omega}^{2}\right) \\
+\int_{0}^{t}(K D, D)_{\Omega}+\mu_{1} \int_{0}^{t}\left\|v_{x}\right\|_{2, \Omega}^{2}=\int_{Q_{T}} f v .
\end{gathered}
$$

Consequently,

$$
\begin{gathered}
\frac{1}{2}\left(\left\|v_{t}\right\|_{2, \Omega}^{2}-\|v(0)\|_{2, \Omega}^{2}\right)+\frac{1}{2} \mu\left(\left\|v_{x}(t)\right\|_{2, \Omega}^{2}-\left\|v_{x}(0)\right\|_{2, \Omega}^{2}\right) \\
\leq \int_{0}^{t}|(f, v)| \leq\|f\|_{2,1}\|v\|_{2, \infty} .
\end{gathered}
$$

Maximization of the left-hand side implies

$$
\frac{1}{2}\|v\|_{2, \Omega}^{2} \leq\|v\|_{2, \infty}\|f\|_{2,1}+\frac{1}{2}\|v(0)\|_{2, \Omega}^{2}+\frac{1}{2} \mu\left\|v_{x}(0)\right\|_{2, \Omega}^{2} .
$$


Thus the norm $\|v\|_{2, \Omega}$ is estimated by

$$
\|v\|_{2, \Omega} \leq\|f\|_{2,1}+\left(\|f\|_{2,1}^{2}+\|v(0)\|_{2, \Omega}^{2}+\mu\left\|v_{x}(0)\right\|_{2, \Omega}^{2}\right)^{1 / 2}:=c_{6}(t) .
$$

From (4.6) and (4.8), we deduce that

$$
\begin{aligned}
\|v(t)\|_{2, \Omega}^{2}+\mu\left\|v_{x}(t)\right\|_{2, \Omega}^{2} & \leq 2\|v\|_{2, \Omega}\|f\|_{2,1}+\|v(0)\|_{2, \Omega}^{2}+\mu\left\|v_{x}(0)\right\|_{2, \Omega}^{2} \\
& \leq 2 c_{6}\|f\|_{2,1}+\|v(0)\|_{2, \Omega}^{2}+\mu\left\|v_{x}(0)\right\|_{2, \Omega}^{2}:=c_{7}(t) .
\end{aligned}
$$

Due to (4.5), we can moreover estimate the norm $\int_{0}^{t}\left\|v_{x}\right\|_{2, \Omega}^{2}$ :

$$
\begin{aligned}
\| v(t) & \left\|_{2, \Omega}^{2}+\mu\right\| v_{x}(t)\left\|_{2, \Omega}^{2}+2 \mu_{1} \int_{0}^{t}\right\| v_{x} \|_{2, \Omega}^{2} \\
& \leq\|v(0)\|_{2, \Omega}^{2}+\mu\left\|v_{x}(0)\right\|_{2, \Omega}^{2}+2\|f\|_{2,1}\|v\|_{2, \infty} \\
& \leq\|v(0)\|_{2, \Omega}^{2}+\mu\left\|v_{x}(0)\right\|_{2, \Omega}^{2}+2 c_{6}\|f\|_{2,1}:=c_{8}(t) .
\end{aligned}
$$

Now we differentiate the first equation of system (4.1) with respect to $t$, multiply by $v_{t}$, and integrate over $\Omega$ :

$$
\begin{gathered}
\frac{1}{2} \frac{d}{d t}\left\|v_{t}\right\|_{2, \Omega}^{2}+\mu_{1}\left\|v_{x t}\right\|_{2, \Omega}^{2}+\int_{\Omega}\left(\frac{\partial}{\partial t}(\mathbf{K} D)\right):(\nabla v)_{t} \\
+\int_{\Omega} v_{k t} v_{x_{k}} v_{t}+\frac{1}{2} \mu \frac{d}{d t}\left\|v_{x t}\right\|_{2, \Omega}^{2}=\left(f_{t}, v_{t}\right)_{\Omega} .
\end{gathered}
$$

Using similar estimates for $\int_{\Omega} v_{k t} v_{x_{k}} v_{t}, \int_{\Omega}(\partial / \partial t)(\mathbf{K} D)(\nabla v)_{t}$ and $\left(f_{t}, v_{t}\right)_{\Omega}$ as in (3.21) and applying the Young inequality (2.9), we arrive at the following relation:

$$
\begin{aligned}
\frac{1}{2} \frac{d}{d t}\left\|v_{t}\right\|_{2, \Omega}^{2}+\mu_{1}\left\|v_{x t}\right\|_{2, \Omega}^{2}+\frac{1}{2} \frac{d}{d t} \mu\left\|v_{x t}\right\|_{2, \Omega}^{2} \\
\leq\left(\theta\left\|f_{t}\right\|_{2, \Omega}+c_{\theta}\left\|f_{t}\right\|_{2, \Omega}\left\|v_{t}\right\|_{2, \Omega}^{2}\right)+\left(\frac{4}{3}\right)^{3 / 4}\left(\kappa_{3}\left\|v_{x t}\right\|_{2, \Omega}^{2}+c_{\kappa_{3}}\left\|v_{x}\right\|_{2, \Omega}^{4}\left\|v_{t}\right\|_{2, \Omega}^{2}\right) \\
\quad+\left(c_{\kappa_{1}}\left\|\frac{\partial}{\partial t}(\mathbf{K} D)\right\|_{2, \Omega}^{2}+\kappa_{1}\left\|v_{x t}\right\|_{2, \Omega}^{2}\right) .
\end{aligned}
$$

Define the constants $\theta, \kappa_{i}$ :

$$
\left(\frac{4}{3}\right)^{3 / 4} \kappa_{3}=\frac{\mu_{1}}{4}, \quad \kappa_{1}=\frac{\mu_{1}}{4}, \quad c_{\kappa_{1}}=\frac{1}{\mu_{1}} ; \quad c_{\kappa_{3}}=\frac{12}{\mu_{1}^{3}}, \quad \theta=c_{\theta}=\frac{1}{2} .
$$

We obtain the estimate

$$
\begin{aligned}
\frac{1}{2} \frac{d}{d t}\left\|v_{t}\right\|_{2, \Omega}^{2}+\frac{1}{2} \mu \frac{d}{d t}\left\|v_{x t}\right\|_{2, \Omega}^{2} \leq & \left(\frac{1}{2}\left\|f_{t}\right\|_{2, \Omega}+\frac{1}{2}\left\|f_{t}\right\|_{2, \Omega}\left\|v_{t}\right\|_{2, \Omega}^{2}\right) \\
& +\frac{4^{7 / 4} 3^{1 / 4}}{\mu_{1}^{3}}\left\|v_{x}\right\|_{2, \Omega}^{4}\left\|v_{t}\right\|_{2, \Omega}^{2}+\frac{1}{\mu_{1}}\left\|\frac{\partial}{\partial t}(\mathbf{K} D)\right\|_{2, \Omega}^{2} .
\end{aligned}
$$


74 Boundary value problems for linear viscoelastic fluids

We abbreviate $\zeta=\left\|v_{t}\right\|_{2, \Omega}^{2}+\mu\left\|v_{x t}\right\|_{2, \Omega}^{2}, c_{\mu_{1}}=2\left(4^{7 / 4} 3^{1 / 4} / \mu_{1}^{3}\right)$. Then,

$$
\begin{aligned}
\frac{d}{d t} \zeta & \leq\left\|f_{t}\right\|_{2, \Omega}+\left\|f_{t}\right\|_{2, \Omega}\left\|v_{t}\right\|_{2, \Omega}^{2}+c_{\mu_{1}}\left\|v_{x}\right\|_{2, \Omega}^{4}\left\|v_{t}\right\|_{2, \Omega}^{2}+\frac{2}{\mu}\left\|\frac{\partial}{\partial t}(\mathbf{K} D)\right\|_{2, \Omega}^{2} \\
& \leq\left(\left\|f_{t}\right\|_{2, \Omega}+c_{\mu_{1}}\left\|v_{x}\right\|_{2, \Omega}^{4}\right) \zeta+\left(\left\|f_{t}\right\|_{2, \Omega}+\frac{2}{\mu_{1}}\left\|\frac{\partial}{\partial t}(\mathbf{K} D)\right\|_{2, \Omega}^{2}\right) .
\end{aligned}
$$

By the Gronwall lemma

$$
\begin{aligned}
& \zeta \leq \exp \left(\int_{0}^{t}\left(\left\|f_{t}\right\|_{2, \Omega}+c_{\mu_{1}}\left\|v_{x}\right\|_{2, \Omega}^{4}\right)\right) \\
& \cdot\left[\left\|v_{t}(0)\right\|_{2, \Omega}^{2}+\mu\left\|v_{x t}(0)\right\|_{2, \Omega}^{2}+\int_{0}^{t}\left(\left\|f_{t}\right\|_{2, \Omega}+\frac{2}{\mu_{1}}\left\|\frac{\partial}{\partial t}(\mathbf{K} D)\right\|_{2, \Omega}^{2}\right)\right] .
\end{aligned}
$$

Thus the following estimate is true:

$$
\begin{aligned}
\left\|v_{t}\right\|_{2, \Omega}^{2} & +\mu\left\|v_{x t}\right\|_{2, \Omega}^{2} \\
\leq & \exp \left(\int_{0}^{t}\left(\left\|f_{t}\right\|_{2, \Omega}+c_{\mu_{1}} \frac{c_{7}(t)}{\mu}\right)\right) \\
& \cdot\left[\left\|v_{t}(0)\right\|_{2, \Omega}^{2}+\mu\left\|v_{x t}(0)\right\|_{2, \Omega}^{2}+\int_{0}^{t}\left\|f_{t}\right\|_{2, \Omega}+\frac{1}{\mu_{1}^{2}} c_{1}^{2} c_{8}\right]:=c_{9}(t) .
\end{aligned}
$$

4.3. Existence theorem. The estimates (4.9),(4.10), and (4.17) obtained in the preceding subsection imply the existence of the solution for the initial boundary value problem (4.1)-(4.2).

TheOREM 4.1. Let $\Omega$ be an arbitrary bounded domain in $\mathbb{R}^{3}, \partial \Omega \in \mathbb{C}^{0,1}$,

$$
f, f_{t} \in \mathbf{L}_{2,1}\left(Q_{T}\right), \quad 0<T \leq \infty, \quad v_{0} \in \mathbf{W}_{2}^{2}(\Omega) \cap \mathbf{J}^{0}(\Omega) .
$$

Assume that operator $\mathbf{K}$ satisfies conditions (3.2)-(3.4). Then problem (4.1), (4.2) has a generalized solution $v$ such that

$$
v, v_{t} \in \mathbf{L}_{\infty}\left(0, T ; \mathbf{J}^{0}(\Omega)\right), \quad v_{x}, v_{x t} \in \mathbf{L}_{2}\left(Q_{T}\right) \cap \mathbf{L}_{\infty}\left(0, T ; \mathbf{J}^{0}(\Omega)\right),
$$

which satisfies the following estimates:

$$
\begin{gathered}
\|v\|_{2, \infty}^{2}+\mu\left\|v_{x}\right\|_{2, \infty}^{2}+2 \mu_{1} \int_{0}^{t}\left\|v_{x}\right\|_{2, \Omega}^{2} \leq c_{8}\left(\|f\|_{2,1} ;\|v(0)\|_{2, \Omega} ;\left\|v_{x}(0)\right\|_{2, \Omega}\right), \\
\left\|v_{t}\right\|_{2, \infty}^{2}+\mu\left\|v_{x t}\right\|_{2, \Omega}^{2} \leq c_{9}\left(\left\|f_{t}\right\|_{2,1} ;\left\|v_{t}(0)\right\|_{2, \Omega} ;\left\|v_{x t}(0)\right\| \|_{2, \Omega}\right)
\end{gathered}
$$

furthermore, $c_{8}(t), c_{9}(t)$ tend to some constants $c_{8}, c_{9} \leq \infty$ when $t \rightarrow \infty$.

The proof of this theorem is similar to the proof of Theorem 3.1. 
4.4. Equations of motion of the linear viscoelastic Kelvin-Voight fluids. Now we show that the system of equations describing the motion of a linear viscoelastic Kelvin-Voight fluid belongs to the considered class.

The system of equations describing the Kelvin-Voight flow can be obtained from the system of equations of incompressible media (3.36) when the deviator of the stress tensor $\sigma$ and the tensor of velocity deformations $D$ satisfy the relation (3.37). We assume, that $M=L+1, L=0,1, \ldots$. Transforming this relation in a similar way as in the previous section, we obtain an expression for the tensor $\sigma$

$$
\sigma=\mu \frac{\partial D}{\partial t}+\mu_{1} D+\sum_{s=1}^{L} \beta_{s}^{(k)} \int_{0}^{t} e^{-\alpha_{s}(t-\tau)} D d \tau
$$

where $\left(-\alpha_{s}\right)$ are the roots of polynomial $Q(p)$ and the coefficients $\beta_{s}^{(k)}$ are supposed to be positive

$$
\beta_{s}^{(k)}=P_{k}\left(\alpha_{s}\right)\left[Q^{\prime}\left(\alpha_{s}\right)\right]^{-1}>0
$$

$P_{k}$ are the polynomials

$$
\begin{gathered}
P_{k}(p)=\nu-\mu p-\mu_{1}+\sum_{l=1}^{L-1}\left(\kappa_{l}-\mu_{1} \lambda_{l}-\mu \lambda_{l-1}\right) p^{l}, \\
\mu=\kappa_{L+1} \lambda_{L}^{-1}, \quad \mu_{1}=\left(\kappa_{l}-\mu \lambda_{L}\right) \lambda_{L}^{-1}, \quad \lambda_{0}=0 .
\end{gathered}
$$

We assume again that the coefficients $-\alpha_{s}$ are single, that is, $Q^{\prime}\left(-\alpha_{s}\right) \neq 0, l=1,2, \ldots, L$, real valued and negative. Thus the equations of motion of the Kelvin-Voight fluids take the form

$$
\frac{\partial v}{\partial t}+v_{k} \frac{\partial v}{\partial x_{k}}-\mu_{1} \Delta v-\mu \frac{\partial \Delta v}{\partial t}-\sum_{s=1}^{L} \beta_{s}^{(k)} \int_{0}^{t} e^{-\alpha_{s}(t-\tau)} \Delta v d \tau+\operatorname{grad} p=f, \quad \operatorname{div} v=0
$$

Obviously, this system is of type (4.1) with the operator

$$
\mathbf{K}_{k} v=\int_{0}^{t} K(t-\tau) v(x, \tau) d \tau
$$

where the kernel $K$ has the form

$$
K(t)=\sum_{s=1}^{L} \beta_{s}^{(k)} e^{-\alpha_{s} t}
$$

The operator $\mathbf{K}_{k}$ has a similar form as the operator $\mathbf{K}_{o}$ from the previous section. So it possesses the properties (3.2)-(3.4). Thus the existence theorem is valid also for the systems describing motions of the Kelvin-Voight fluids. 


\section{Equations of general type}

5.1. Formulation of the problem. In this section, we consider rheological equations of more general type (1.7). Suppose that the operator $\mathbf{K}$ maps $\mathbf{L}_{2}\left(Q_{T}\right)$ into $\mathbf{L}_{2}\left(Q_{T}\right)$ and satisfies conditions (3.2), (3.3). These conditions are fulfilled for rheological equations of any non-Newtonian (and Newtonian) fluid.

Substituting (1.7) into the equation of motion of incompressible fluid (1.1), we obtain the system

$$
\frac{\partial v}{\partial t}+v_{k} \frac{\partial v}{\partial x_{k}}-\operatorname{div} \mathbf{K} D+\operatorname{grad} p(x, t)=f(x, t) \quad \operatorname{div} v(x, t)=0 .
$$

At first, we consider an initial boundary value problem with periodic boundary conditions. Let $\Omega_{R}=[-R, R] \times[-R, R]$ be a square in $\mathbb{R}^{2}$. System (5.1) is considered in the cylinder $Q_{T}=\Omega_{R} \times[0, T]$. Suppose that the functions $v$ and $p$ satisfy periodic boundary conditions on the sides of the square. We formulate them for the function $v$ :

$$
\left.v\right|_{x_{1}=-R}=\left.v\right|_{x_{1}=R},\left.\quad v\right|_{x_{2}=-R}=\left.v\right|_{x_{2}=R},\left.\quad v_{x_{1}}\right|_{x_{1}=-R}=\left.v_{x_{1}}\right|_{x_{1}=R},\left.\quad v_{x_{2}}\right|_{x_{2}=-R}=\left.v_{x_{2}}\right|_{x_{2}=R} .
$$

Moreover, the function $v$ satisfies the initial condition

$$
\left.v\right|_{t=0}=v_{0}(x), \quad x \in \Omega_{R} .
$$

Multiply the system (5.1) by $v$ and integrate over $\Omega$. After integrating by parts, we get

$$
\frac{1}{2} \frac{d}{d t}\|v\|_{2, \Omega}^{2}+\int_{\Omega}((\mathbf{K} D): D)=\int_{\Omega} f v .
$$

Integration with respect to $t$ implies the identity

$$
\frac{1}{2}\left(\|v(t)\|_{2, \Omega}^{2}-\|v(0)\|_{2, \Omega}^{2}\right)+\int_{Q_{T}}((\mathbf{K} D): D)=\int_{Q_{T}} f v .
$$

Proceeding as in Section 3.2 and using the nonnegativity of the term $\int_{Q_{T}}((K D): D)$, we get a priori estimate for the norm of $v$

$$
\|v\|_{2, \infty}=\int_{0}^{t}\|f\|_{2, \Omega}+\sqrt{\left(\int_{0}^{t}\|f\|_{2, \Omega}\right)^{2}+\|v(0)\|_{2, \Omega}^{2}} .
$$

We prove the existence of at least a one weak solution from the space $\mathbf{L}_{\infty}\left(0, T ; \hat{\mathbf{J}}_{2}^{1}(\Omega)\right)$ which satisfies the integral identity

$$
\int_{Q_{T}}\left(-v \phi_{t}-v_{k} v \phi_{x_{k}}+(\mathbf{K} D) \nabla \phi\right) d x d t+\left.\int_{\Omega_{R}} v \phi\right|_{t=T} d x-\left.\int_{\Omega_{R}} v_{0} \phi\right|_{t=0} d x=\int_{Q_{T}} f \phi
$$

for any solenoidal periodic $\phi$ such that $\phi_{x}, \phi_{x t} \in \mathbf{L}_{2}\left(Q_{T}\right)$.

We project system (5.1) onto the space of divergence-free vector fields and annihilate the term $\operatorname{grad} p$, find a weak solution of obtained boundary value problem. When the velocity is found, the pressure may be found from system (5.1). 
5.2. A priori estimates of the solutions. Now we assume that the operator $\mathbf{K}$ possesses the following property:

$$
-(\operatorname{rot} \operatorname{div}(\mathbf{K} D), \operatorname{rot} v) \geq 0
$$

The operation rot is understood as the scalar function in the space $\mathbb{R}^{2}$

$$
\operatorname{rot} u=\frac{\partial u_{2}}{\partial x_{1}}-\frac{\partial u_{1}}{\partial x_{2}}
$$

If the operators $\mathbf{K}$ and $\partial / \partial x_{k}, k=1,2$ commute then condition (5.8) is a corollary of (3.3). This condition (5.8) is fulfilled, for instance, in the case when the operator $\mathbf{K}$ is integral operator of the form (1.9). Then the condition of positiveness (3.3) may be improved in the following manner. We may suppose that the kernel $K(t)$ of the operator $\mathbf{K}$ is a positive definite function. It means that the matrix $\left\{K\left(t_{i}-t_{j}\right)\right\}_{i, j=1}^{N}$ is positive in $\mathbb{C}^{N}$ for every $N$ and for any $t_{1}, \ldots, t_{N} \in \mathbb{R}^{N}$.

We estimate the space derivative of the function $v$.

Consider the stream function $v=\left(-\psi_{x_{2}}, \psi_{x_{1}}\right)$. To obtain the following relation, we apply the operator rot to both parts of the first equation of system (5.1). Then it holds

$$
\Delta \psi_{t}-\operatorname{rot} \operatorname{div}(\mathbf{K} D)=\psi_{x_{1}} \Delta \psi_{x_{2}}-\psi_{x_{2}} \Delta \psi_{x_{1}}+\varphi
$$

where $\varphi(x, t)=\left(\partial f_{2} / \partial x_{1}\right)-\left(\partial f_{1} / \partial x_{2}\right)$. Moreover, we suppose that the function $\psi$ satisfies periodic boundary conditions. Let all the first-order and the second-order space derivatives of the function $\psi$ be periodic functions in $\Omega_{R}$.

The initial condition takes the form

$$
\left.\psi\right|_{t=0}=b(x),
$$

where $b_{x_{1}}=-v_{02}, b_{x_{2}}=v_{01}$. ( $v_{i}$ is the $i$-th coordinate of the function $v$.)

If we multiply equation (5.10) by $\Delta \psi$ in the space $\mathbf{L}_{2}\left(\Omega_{R}\right)$, we arrive at the identity

$$
\frac{1}{2} \frac{d}{d t}\left\|\Delta \psi_{t}\right\|_{2, \Omega}^{2}-(\operatorname{rot} \operatorname{div}(\mathbf{K} D), \operatorname{rot} v)=(\varphi, \Delta \psi)
$$

This relation is similar to relation (3.9). Thus we find an estimate similar to estimate (3.13) for the function $\Delta \psi$, namely,

$$
\|\Delta \psi\|_{2, \infty} \leq\|\varphi\|_{2,1}+\sqrt{\|\varphi\|_{2,1}^{2}+\|\Delta \psi(0)\|_{2, \Omega}^{2}} .
$$

Since $\left\|v_{x}\right\|_{2, \infty} \leq\|\Delta \psi\|_{2, \infty},(5.13)$ implies a further estimate

$$
\left\|v_{x}\right\|_{2, \infty} \leq c\left(\|\varphi\|_{2,1}+\sqrt{\|\varphi\|_{2,1}^{2}+\|\Delta \psi(0)\|_{2}^{2}}\right) .
$$


5.3. Existence of the solution. To prove the existence of a weak solution, we apply Galerkin's method with the system of eigenfunctions of the Stokes problem

$$
\begin{aligned}
& -\Delta v=\lambda v, \quad \operatorname{div} v=0, \\
& v \in \widehat{\mathbf{J}}_{2}^{1}(\Omega) \cap \mathbf{W}_{2}^{2}(\Omega)=\widehat{\mathbf{J}}_{2}^{2}(\Omega)
\end{aligned}
$$

as basis functions. By [8] the functions $\left\{\varphi^{l}\right\}_{l=0}^{\infty}$ form an orthonormal system in the space $\hat{\mathbf{J}}_{2}^{0}(\Omega)$, and so it is the case in the spaces $\widehat{\mathbf{J}}_{2}^{1}(\Omega)$ and $\hat{\mathbf{J}}_{2}^{2}(\Omega)$.

The Galerkin approximations are defined as

$$
v^{n}(x, t)=\sum_{l=0}^{n} c_{l n}(t) \varphi^{l}(x),
$$

where the coefficients $c_{l n}(t)$ are determined from the system

$$
\frac{d}{d t}\left(v_{n}, \varphi^{l}\right)+\left(\mathbf{K} v_{x}^{n}, \varphi_{x}^{l}\right)+\left(v_{k}^{n} v_{x_{k}}^{n}, \varphi^{l}\right)=\left(f, \varphi^{l}\right)
$$

with initial conditions

$$
c_{l n}(0)=\left(v_{0}, \varphi^{l}\right), \quad l=0, \ldots, n .
$$

The approximations $v^{n}$ satisfy the same identities

$$
\frac{1}{2} \frac{d}{d t}\left\|v^{n}\right\|_{2, \Omega}^{2}+\int_{\Omega}\left(\left(\mathbf{K} D\left(v^{n}\right)\right): D\left(v^{n}\right)\right)=\int_{\Omega} f v^{n}
$$

as the exact solutions. So estimates (3.13) and (5.14) are true for them. And a majorant is independent of $n$. This permits to pass to the limit and prove the next theorem.

THEOREM 5.1. Let the following functions be given:

$$
f \in \mathbf{L}_{2,1}\left(Q_{T}\right), \quad f_{x} \in \mathbf{L}_{2,1}\left(Q_{T}\right), \quad v_{0} \in \hat{\mathbf{J}}_{2}^{1}\left(\Omega_{R}\right) .
$$

Then the initial boundary value problem (5.1), (5.2), (5.3) admits a generalized solution

$$
v \in \mathbf{L}_{\infty}\left(0, T ; \hat{\mathbf{J}}_{2}^{1}\left(\Omega_{R}\right)\right)
$$

which is global in $t$. The solution $v$ satisfies estimates (3.13) and (5.14).

The corollary of this theorem consists of the existence of a solution (5.21) for the systems of equations of general linear viscoelastic liquids with rheological equation of the type (1.9). All the conditions (3.2), (3.3), and (5.8) are fulfilled for these equations.

5.4. The Cauchy problem. From Theorem 3.1, it follows that the Cauchy problem is also solvable.

Theorem 5.2. Let

$$
f \in \mathbf{L}_{2,1}\left(\mathbb{R}^{2} \times[0, T]\right), \quad f_{x} \in \mathbf{L}_{2,1}\left(\mathbb{R}^{2} \times[0, T]\right), \quad v_{0} \in \mathbf{J}_{2}^{1}\left(\mathbb{R}^{2}\right),
$$


where $\mathbf{J}_{2}^{1}\left(\mathbb{R}^{2}\right)$ is a space of solenoidal vector fields with finite norm $\|u\|_{2}+\left\|u_{x}\right\|_{2}$. Then there exists a generalized solution of system (5.1) which satisfies the initial Cauchy condition

$$
v(x, 0)=v_{0}(x), \quad x \in \mathbb{R}^{2} .
$$

This solution belongs to $\mathbf{L}_{\infty}\left(0, T ; \mathbf{J}_{2}^{1}\left(\mathbb{R}^{2}\right)\right)$ and satisfies estimates (3.13) and (5.14).

This theorem is also true for general linear viscoelastic liquids.

Proof. The theorem can be proved in the following way. We choose the sequence of extending domains $\Omega_{R_{n}}, R_{n} \rightarrow \infty$, and construct the solution $v_{n}$ of periodic boundary value problem in every domain. The norms $\left\|v^{n}\right\|_{2, \infty},\left\|v_{x}^{n}\right\|_{2, \infty}$ of all solutions $v^{n}$ are bounded by a majorant, which is independent of the domain. If we use compactly supported test functions, then we can pass to the limit in the identities (5.7) for functions $v^{n}$.

5.5. Equations of motion of the Maxwell fluids. The motion of the Maxwell fluid is determined by the relation (3.37), together with $M=L-1, L=1,2, \ldots$. Proceeding exactly as in the previous sections, we can represent the deviator of the stress tensor $\sigma$ in the form

$$
\sigma=\sum_{s=1}^{L} \beta_{s}^{(m)} \int_{0}^{t} e^{-\alpha_{s}(t-\tau)} D(x, \tau) d \tau,
$$

where

$$
\beta_{s}^{(m)}=P_{m}\left(\alpha_{s}\right)\left[Q^{\prime}\left(\alpha_{s}\right)\right]^{-1},
$$

and the polynomial $P_{m}$ is given by

$$
P_{m}(p)=v+\sum_{l=1}^{L-1} \kappa_{l} p^{l}
$$

The flow of the Maxwell fluid is described by the system (5.1) with the operator $\mathbf{K}_{m}$

$$
\mathbf{K}_{m}=\sum_{s=1}^{L} \beta_{s}^{(m)} e^{-\alpha_{s} t} .
$$

It is evident that the operator $\mathbf{K}_{m}$ possesses properties (3.2), (3.3), and (5.8), and therefore the existence theorem is also valid for the Maxwell fluids.

\section{Acknowledgment}

The author thanks Professor Dr. S. Pickenhain and Dr. M. Wagner for useful discussions.

\section{References}

[1] G. Astarita, G. Marucci, Principles of Non-Newtonian Fluid Mechanics, McGraw-Hill, London, 1974.

[2] R. B. Bird, R. C. Armstrong, and O. Hassanger, Dynamics of Polymeric Liquids, I, John Wiley \& Sons, New York, 1987. 
[3] G. J. Creus, Viscoelasticity-Basic Theory and Applications to Concrete Structures, Lecture Notes in Engineering, vol. 16, Springer-Verlag, Berlin, 1986.

[4] M. Fabrizio and J. M. Golden, Maximum and minimum free energies for a linear viscoelastic material, Quart. Appl. Math. 60 (2002), no. 2, 341-381.

[5] G. Gentili, Maximum recoverable work, minimum free energy and state space in linear viscoelasticity, Quart. Appl. Math. 60 (2002), no. 1, 153-182.

[6] J. M. Golden and G. A. C. Graham, Boundary Value Problems in Linear Viscoelasticity, SpringerVerlag, Berlin, 1988.

[7] J. W. Goodwin and R. W. Hughes, Rheology for Chemists: An Introduction, Royal Society of Chemistry, Cambridge, 2000.

[8] O. A. Ladyzhenskaya, The Mathematical Theory of Viscous Incompressible Flow, Mathematics and its Applications, vol. 2, Gordon and Breach Science Publishers, New York, 1969.

[9] A. Matei, V. V. Motreanu, and M. Sofonea, On the Signorini frictionless contact problem for linear viscoelastic materials, Appl. Anal. 80 (2001), no. 1-2, 177-199.

[10] A. P. Oskolkov, Certain model nonstationary systems in the theory of non-Newtonian fluids, Tr. Mat. Inst. Steklova 127 (1975), 32-57 (Russian).

[11] On the theory of Maxwell fluids, Zap. Nauchn. Sem. Leningrad. Otdel. Mat. Inst. Steklov. (LOMI) 101 (1981), 119-127 (Russian).

[12] _ Initial-boundary value problems for equations of motion of Kelvin-Voight fluids and Oldroyd fluids, Tr. Mat. Inst. Steklova 179 (1988), 126-164 (Russian).

[13] F. R. Schwarzl, Polymermechanik, Springer-Verlag, Berlin, 1990.

[14] N. W. Tschoegl, The Phenomenological Theory of Linear Viscoelastic Behavior, Springer-Verlag, Berlin, 1989.

N. A. Karazeeva: Petersburg Department, V. A. Steklov Institute of Mathematics, 27 Fontanka, St. Petersburg 191011, Russia

E-mail address: karazeev@pdmi.ras.ru 


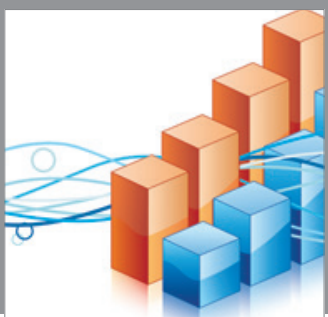

Advances in

Operations Research

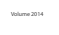

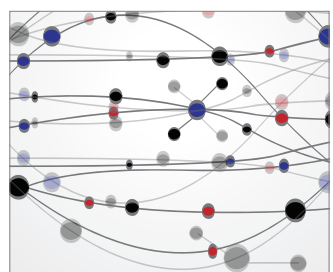

\section{The Scientific} World Journal
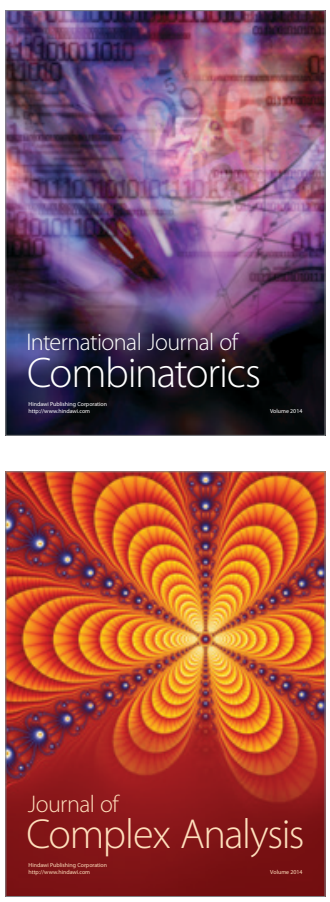

International Journal of

Mathematics and

Mathematical

Sciences
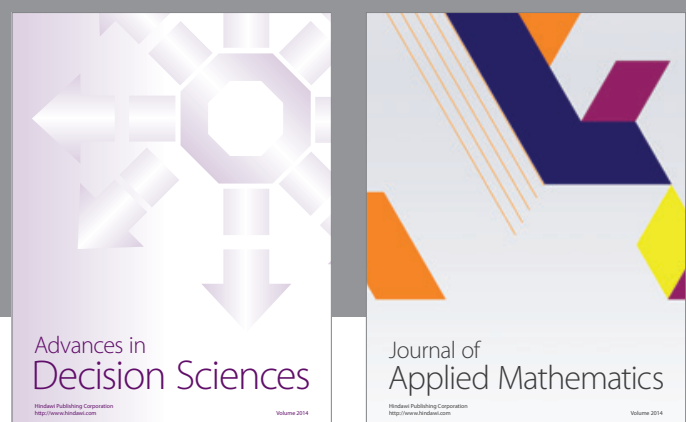

Journal of

Applied Mathematics
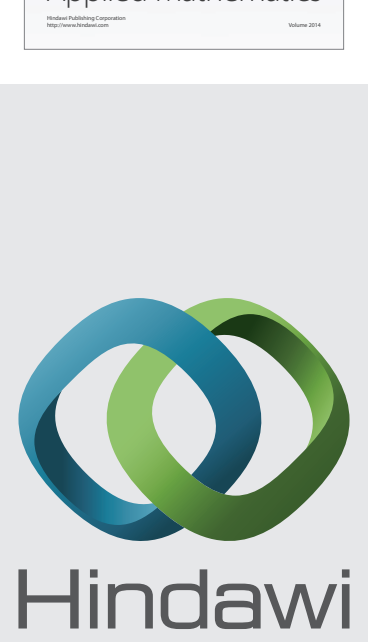

Submit your manuscripts at http://www.hindawi.com
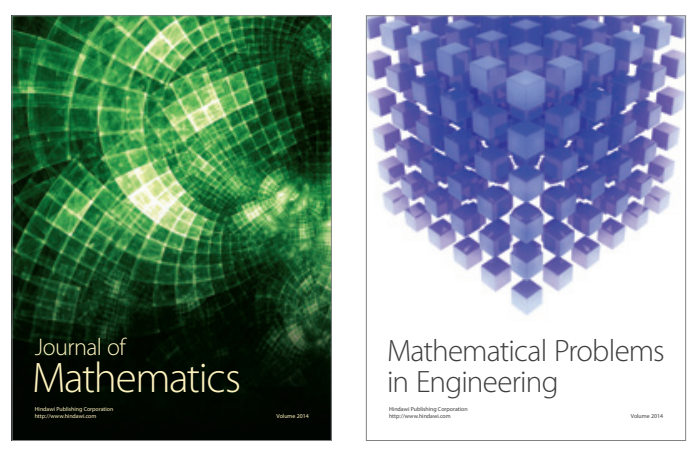

Mathematical Problems in Engineering
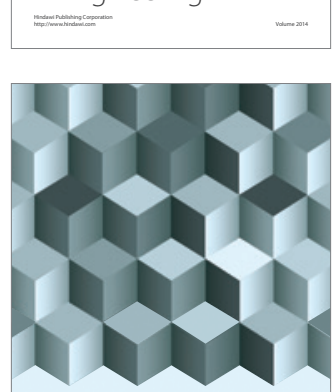

Journal of

Function Spaces
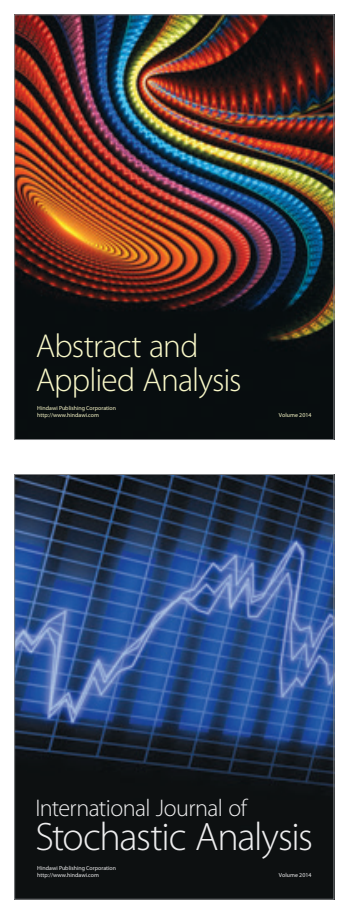

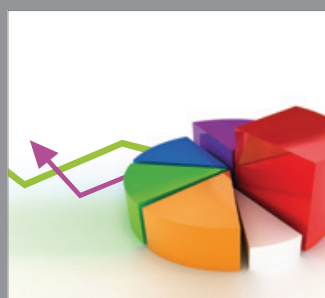

ournal of

Probability and Statistics

Promensencen
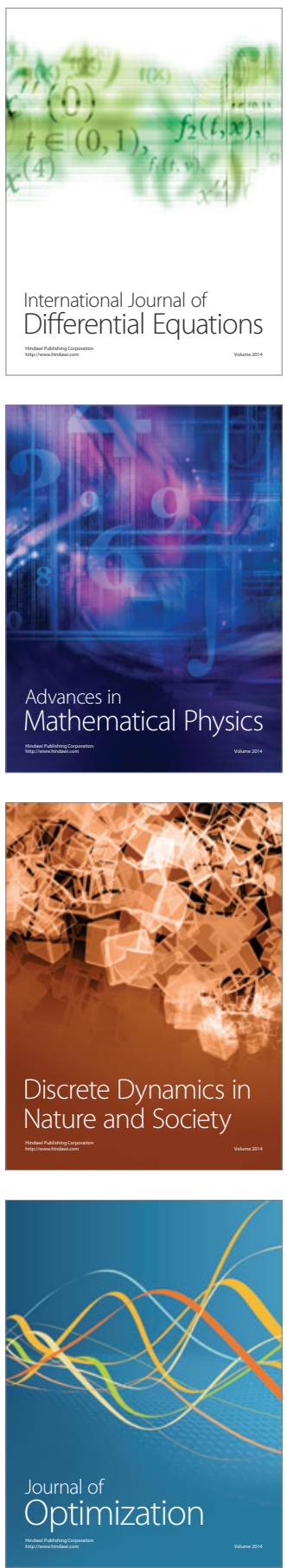